\title{
CÓMO HACER RAZONES CON NORMAS O CÓMO CONVERTIR NORMAS EN RAZONES O CÓMO DESENREDAR ESTE LÍO
}

\author{
Rolando Tamayo y Salmorán \\ Universidad Nacional Autónoma de México (México) \\ Seguro no entiendo la idea de razón para \\ actuar y me pregunto si algún otro la entiende. ${ }^{2}$
} Philippa Foot

\section{Introducción}

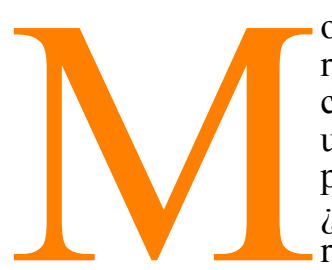

ovidos por la fascinación que irradia la argumentación y el razonamiento jurídicos, algunos autores han llegado al extremo de caracterizar a las normas jurídicas como razones. ${ }^{3} \mathrm{Al}$ hacerlo cometen un tipo de falacia naturalista. ${ }^{4}$ Las normas (como cualquier cosa) pueden ser tratadas como razones (justificaciones) para la acción, pero ¿qué acaso ese hecho no permite pensar que las normas sean meras razones?

Esta idea gira alrededor de una concepción ampliamente compartida sobre la racionalidad. Existe la necesidad por la racionalidad. Y así, si las normas son razones, entonces la racionalidad en el derecho estaría asegurada. Sin embargo, el derecho no es una manifestación inmanente de la racionalidad. Pero, aun si el derecho no es una individuación necesaria de la razón, queda una cuestión pendiente: ¿cómo operaría la racionalidad en el funcionamiento del derecho?

${ }^{1}$ Versión española de mi ensayo: «How to Make Reasons with Norms. Or How to Convert Norms into Reasons. Or How to Disentangle this Mess». En cuanto al título pienso que es completamente ocioso referirme al célebre trabajo de John Austin o al conocido libro de Twinning y Miers. Véase la Bibliografía.

${ }^{2}$ La cita continúa así: «Yo en lo particular me inclino por la idea de que todas esas razones dependen de los intereses del agente o... de sus deseos», en «Reason for Action and Desires» [Postscript] en Foot, Philippa, Virtues and Vices and Other Essays in Moral Philosophy, Oxford, Basil Blackwell, 1978, p. 156.

${ }^{3}$ Prestigiados autores han levantado fuertes objeciones contra la tesis de que las normas sean razones (para la acción). Véase, por ejemplo: Cristina Redondo, Pablo Navarro, Carlos Nino, Javier Ortiz, etc. (Vid. infra: Bibliografia). Algunas de esas objeciones, ciertamente las comparto; sin embargo en este ensayo no abordaré tales objeciones (o no sólo). Mi idea es un poco diferente, intento demostrar que aquellos que defienden esa tesis cometen un tipo de falacia naturalista.

${ }^{4}$ En el sentido del open question argument de George E. Moore (1873-1958). Pienso que el argumento de Moore puede aplicarse similarmente contra el intento de definir normas en términos de alguna cualidad de las razones. (Cf.: Principia Ethica, Cambridge, 1903; Vid.: Baldwin, Thomas. G. E. Moore, Londres, 1990. 
Dos cuestiones han fuertemente intrigado a los seres humanos a través de la historia de la humanidad: “¿qué es?” y “¿qué hacer?”. Las respuestas dadas a estas cuestiones han producido dos diferentes derroteros. Éstos son: la descripción (i.e. la explicación), por un lado, y la prescripción y justificación, por el otro. Este último constituye el campo (por así decirlo) de la razón práctica.

En lo que a las normas se refiere, pienso que, conforme con esta bifurcación, debe darse dos diferentes respuestas al problema.

Una tesis que subyace todo este ensayo es que una cosa es describir normas (i.e. el derecho) como razones (lo que es básicamente erróneo) y una muy distinta es usar normas jurídicas en la argumentación jurídica. El propósito de este ensayo es llamar la atención hacia esta importante diferencia y emprender la búsqueda de respuestas a estos problemas. La respuesta, aquí, es fragmentaria y tentativa.

\section{Normas y razones}

\section{Preliminaria}

Muchos filósofos y teóricos del derecho piensan que los hombres actúan sobre la base de razones. Asimismo, muchos filósofos y teóricos del derecho piensan que cualquier cosa, i.e. cualquier hecho (de aquí en adelante $\mathrm{X}$ ) puede ser una razón para actuar (o una razón para abstenerse de actuar). De esta forma, llover es una razón para usar una gabardina. El problema es que no necesariamente cualquier cosa es una razón para actuar (o para no actuar). Que llueva en Kuala Lumpur, ${ }^{5}$ no es razón para que use gabardina, debido al hecho de que vivo en México.

¿Cómo puedo saber si $\boldsymbol{x}$ es o no es una razón? O ¿qué convierte a $\boldsymbol{x}$ en una razón? ${ }^{6}$ (de aquí en adelante $\boldsymbol{R}$ ). No veo otra respuesta: de actuar.

$\boldsymbol{x}$ es una razón porque cuenta en mi consideración para actuar o para abstenerme

Piénsese, por ejemplo, en algo que ignoro ¿Cómo podría ser esto una razón para mi acción? Por el contrario, si yo tomo algo en (mi) consideración (un evento, un dicho, una instrucción), se convierte en una razón para mí (para mi acción o para mi abstención). Es decir, si en mi deliberación (por así decirlo) tomo en consideración a $\boldsymbol{x}$ para mi acción o para abstenerme de

${ }^{5}$ Capital de Malasia, situada en la parte central de Malasia (Malaya) occidental. La ciudad crece a horcajadas de la confluencia de los ríos Kelang y Gombak en paraje montuoso al oeste del territorio. En 1944 Kuala Lumpur devino el Territorio Federal de Kuala Lumpur. (Vid.: Encyclopaedia Britannica CD, 2.02, Encyclopaedia Britannica Inc., 1995).

${ }^{6}$ Me ocupo sólo de razones para la acción. De ahí que, en el texto, las palabras «para la acción» podrían estar omitidas. 
actuar, entonces $\boldsymbol{x}$ deviene una razón para que yo haga o una razón para que yo omita.

En este momento quiero resaltar que las razones son razones aunque sean razones triviales. No importa qué débiles sean, una vez en mi consideración, son razones.

De conformidad con lo anterior, tenemos que distinguir entre cosas que no son razones (para actuar) y cosas que son razones de poca importancia (para actuar). Si no llueve fuerte, sino sólo chispea y tengo que caminar sólo unos pasos y, además, tengo prisa, entonces la lluvia es una razón, pero una razón a la cual concedo poca importancia. ${ }^{7} \mathrm{Sin}$ embargo, el hecho de que llueva en Kuala Lumpur no es una razón de poco peso, más bien no es en absoluto una razón. De la misma manera, si la contaminación es mi preocupación principal, el hecho de que la atmósfera de Venus tenga un alto contenido de ácido sulfúrico ${ }^{8}$ no es razón para abstenerme de hacer ejercicios aeróbicos y quedarme en casa.

Las razones de poca importancia, sin importar qué poca importancia tengan, son siempre tomadas en cuenta, el agente (de aquí en adelante ) efectivamente las toma en consideración. El agente considera tanto a las razones importantes como a las menos importantes. Las cosas que no tomo en consideración no son razones para mis acciones ni para mis omisiones. Dicho brevemente: las cosas que no tomo en consideración no son razones para mis acciones. De esta forma tenemos:

$$
m=\{x, x, x, x \ldots\}
$$

Donde $x$ es cualquier cosa que ocurre en el Mundo. Y

$$
\mathbb{Q}=\{\boldsymbol{R}, \boldsymbol{R}, \boldsymbol{R}, \boldsymbol{R}, \boldsymbol{R} \ldots\}
$$

Donde $\boldsymbol{R}$ es cualquier cosa que se convierte en razón. $\boldsymbol{x}$ se convierte en $\boldsymbol{R}$ si ciertas condiciones son satisfechas. Así:

$$
a \subset m
$$

\section{Una nota sobre racionalidad}

Muchos autores comparten la idea de que «tomar en cuenta», «evaluar», o «hacer un balance», de razones es pensar racionalmente. Asimismo, piensan que actuar sobre la base de la razón exitosa (la razón que vence otras razones) es actuar racionalmente. Al menos, esto parece satisfacer el paradigma: el silogismo práctico. Aristóteles sostiene que actuar sobre la base de cierta razón es la conclusión de un silogismo práctico. De esta for-

${ }^{7}$ De hecho, es superada, consideradas todas las cosas, por una razón de más peso, o bien, vencida por otras razones (combinadas).

8 «Venus se encuentra completamente envuelto por una densa capa de nubes compuestas básicamente de gotas de ácido sulfúrico concentrado...» (Encyclopaedia Britannica CD. 2.02, Encyclopaedia Britannica, Inc., 1995). 
ma, la acción del agente es, en algún sentido, similar a las conclusiones en lógica. ${ }^{9}$ Ésta parece ser la postura de Joseph Raz. A este respecto dice:

Los enunciados de hechos que son razones para la realización de cierta acción por un cierto agente son las premisas de un argumento cuya conclusión es que hay una razón para que el agente realice la acción o deba realizarla. ${ }^{10}$

\section{Normas como razones para la acción}

a. El problema

¿La norma es una razón para la acción? ¿Puede ser? Ahora bien, si la norma es una razón, ¿qué tipo de razón es? ¿cómo funciona? ${ }^{11}$

La cuestión principal alrededor de este problema es si una norma (de aquí en adelante $N$ ) es una razón sólo porque es una norma. Por supuesto, si suponemos que las normas son razones (y sólo razones) únicamente porque son normas, la cuestión expresada en el título de este ensayo es un sin sentido porque las normas serían ya razones.

b. La regla de reconocimiento de razones

Percibo dos diferentes actitudes que son relevantes para este problema, las cuales pueden, a su vez, subdividirse en dos:

(4) $\boldsymbol{N}_{(S)}$ son $\boldsymbol{R}_{(S)}$ y solamente $\boldsymbol{R}_{(S)}$

Y

(5) $\boldsymbol{N}_{(S)}$ son cierto tipo de $\boldsymbol{R}_{(S)}$

(6) $\boldsymbol{N}_{(S)}$ funcionan como $\boldsymbol{R}_{(S)}$, satisfechas ciertas condiciones,

(7) $\boldsymbol{N}_{(S)}$, funcionan como si fueran las únicas $\boldsymbol{R}_{(S)}$

Ya he señalado lo que se requiere para que $\boldsymbol{x}$ se convierta en $\boldsymbol{R}$. A esta condición (o condiciones) la llamaré: "la regla de reconocimiento de razones". Esta regla no es más que un criterio de identidad de razones (para ser razones). De manera general se puede decir que algo es una razón si y sólo si así es considerada por $A$ (el agente). Anteriormente mencioné (o, más bien, recordé) que muchos filósofos y teóricos del derecho piensan que el siguiente enunciado es verdadero:

${ }^{9}$ Cf.: Nic. Eth., VI, 1140 a 25, 1140 b 20, 1141 b 5 y ss. J. C. Bayón Mohíno da una explicación detallada de este problema ( $C f$.: La normatividad del derecho: deber jurídico y razones para la acción, Madrid, Centro de Estudios Constitucionales. 1991. [Colección El Derecho y la Justicia, 26], p. 55).

${ }^{10}$ Practical Reason and Norms, Hutchinson Co. (Publishers), Ud. 1975, p. 28. Existe versión castellana de Juan Ruiz Manero: Razón práctica y normas, Madrid, Centro de Estudios Constitucionales, 1991. (Colección El Derecho y la Justicia, 27), p. 32.

${ }^{11}$ Adviértase que las cuestiones mencionadas arriba, esto es: “¿Cómo hacer razones con normas?” es del todo diferente a la otra igualmente importante: “cómo hacer normas con razones?”. 
(8) cualquier cosa (i.e. $\boldsymbol{x})$ puede ser una razón.

Es decir, cualquier cosa puede ser una razón para hacer $\boldsymbol{\emptyset}$ cualquier cosa puede ser una razón para no hacer ø. Tiempo tempestuoso, por ejemplo, es una razón para cerrar un puerto a la pesca y a la navegación.

Ciertamente, la mayoría de los autores estarían dispuestos a aceptar (8); sin embargo la mayoría de ellos señalaría que (8), como mencioné anteriormente, no significa que cualquier cosa sea necesariamente una razón para todos, en toda circunstancia. Así, el clima tempestuoso en el Puerto de Murmatisk, ${ }^{12}$ en el noroeste de Rusia (a más de 200 kilómetros al norte del Círculo Polar Ártico), es un hecho que pertenece a la clase de «cualquier cosa». Sin duda, pero no es una razón para usar bufanda a mediados de enero en Buenos Aires.

¿Qué cosas son razones para una específica acción ø? Indisputablemente los hechos (eventos, dichos, instrucciones) que tomo en cuenta para mi acción (ø) o para mi abstención $(\sim \boldsymbol{\emptyset})$. Estoy consciente que ésta es una descripción insuficiente sobre por qué un agente actúa sobre la base de una razón. Estoy dejando a un lado los intrincados problemas de la deliberación (del balance) de razones y de la selección y decisión de la acción. Me limito simplemente a decir un par de palabras sobre por qué algunos hechos entran en la clase de «razones para » (para el agente).

Permítase preguntar: ¿las normas, además de ser normas, pueden ser razones (para actuar)? Ahora bien, si cualquier cosa puede ser una razón entonces las normas (jurídicas), entidades que pertenecen a la clase de «cualquier cosa», pueden ser razones para la acción. Ciertamente, pero para que una norma sea una razón tiene que satisfacer la regla de reconocimiento de razones. De esta manera, si yo no considero (pondero) a la norma $\boldsymbol{N}$ entre las razones para $\boldsymbol{\emptyset}$ o para $\sim \boldsymbol{\emptyset}$, entonces $\boldsymbol{N}$ no es en absoluto una razón para que yo haga $\boldsymbol{\sigma}$ o para que me abstenga de hacer $\boldsymbol{\sigma}$. Y si no obstante, $\boldsymbol{N}$ se me aplica, éste es un hecho que me pasa, no una acción que yo realizo (o decido realizar). ${ }^{13}$

${ }^{12}$ Murmansk. Antiguamente Romanov-na-murmane, puerto marítimo y centro de Murmansk Oblast (Región Administrativa), Rusia. "Murmansk" (probablemente de la palabra suami local: murman, que significa "la orilla de la tierra") es la ciudad más grande del mundo al norte del Círculo Polar (Vid.: Encyclopaedia Britannica, CD, Encyclopaedia Britannica, Inc. 1995).

${ }^{13}$ Esta distinción es fundamental para las proposiciones de razón práctica. Esta distinción ha sido establecida desde la Ética Nicomaquea. De acuerdo con Aristóteles los actos no voluntarios no cuentan para los silogismos prácticos. En este sentido dice: «Las acciones son no voluntarias cuando se hacen bajo coacción y un acto es coaccionado cuando su origen es de fuera, siendo de tal naturaleza que el agente, que es realmente pasivo, en nada contribuye a su realización, por ejemplo, cuando es llevado a un lugar por la fuerza del clima...» (Eth. Nic 1110 a 2-4, Vid. también: 1110 b 10. en Aristotle XIX Nicomachean Ethics, trad de H. Rackham, 
c. Una «escala» sobre normas

El curso del argumento me obliga a decir algo sobre las normas. Sin embargo, aunque voy a tratar con normas (jurídicas) no me siento comprometido a dar cuenta de las diferentes concepciones que los distintos autores han dado sobre las normas. Tengo tres razones para eludir este problema: ${ }^{14}$

(1) Prácticamente todas las teorías sobre normas jurídicas tienen, al menos, una cosa en común: el derecho, i.e. cualquier sistema jurídico, se compone de normas. Las normas jurídicas son las unidades básicas de los órdenes jurídicos. Esto es, el derecho (de aquí en adelante $\boldsymbol{D}$ ) es un conjunto (i.e. un sistema) de normas o, al menos, es el caso de que $N \in \mathbb{D}$, aun cuando pudiera haber otras entidades de $\$$ que no fueran normas. ${ }^{15}$

(2) Los estudiosos y profesionales del derecho ampliamente aceptan que $N$ (cualquier cosa que “ $N$ " signifique) es tal en virtud de que fue establecida por el legislador (de aquí en adelante $\mathfrak{L}$ ). Es decir, $\boldsymbol{N}$ (mandato, directiva o lo que sea) es lo que es, sólo porque ha sido establecida por un acto creador de derecho (un acto de $\mathscr{L}$ ). ${ }^{16}$

(3) Los juristas dogmáticos y los teóricos del derecho «entienden» cómo operan las normas. Una norma puede ser concebida como un mandato, como una directiva, como una regla, et sit cetera. Cualquiera que sea el caso, existe una compartida idea que se encuentra detrás de cualquier con-

Cambridge, Mass., Loeb Classical Library. 1975). La coacción cubre todos los casos en que el agente no es, en absoluto, un agente. (Vid.: MacIntyre, Alasdair. A Short History of Ethics. A History of Moral Philosophy from Homeric Age to Twentieth Century, Simon \& Schuster, 1996. pp. 68-69).

${ }^{14} \mathrm{Sin}$ duda «norma» es uno de los conceptos fundamentales de la dogmática jurídica. Sin embargo, aunque muchos libros se han escrito sobre la «naturaleza» del derecho muy pocos juristas examinan exhaustivamente qué es una norma. (Mutatis mutandi, Vid.: Olivecrona, Karl, The Imperative Element in Law [Der Imperative des Gesetzes], p. 794). De hecho, este concepto es frecuentemente usado sin ser definido en absoluto. (Vid.: Ross, Alf, Directives and Norms, Londres, Routledge \& Kegan Paul, 1968, p. 78). Esta circunstancia no es tan desastrosa si la idea de norma es clara y corresponde a los usos generales y profesionales. La falta de precisión, aunque seria, no es catastrófica; las diferentes formulaciones recuperan, de algún modo, una idea general que básicamente corresponde al conjunto de nuestras intuiciones sobre normas jurídicas, a saber: «prescriben», «regulan la conducta humana», «guían la conducta de los individuos», «provocan comportamientos», et sit cetera). Pero, cualquier cosa que las normas pudieran ser, ellas constituyen las unidades básicas de los órdenes jurídicos.

${ }^{15}$ No obstante este «núcleo teórico compartido» estoy consciente de que los teóricos del derecho no están de acuerdo sobre la naturaleza de los «hechos sociales» en los cuales se apoya un positivista. Vid.: Navarro, Pablo y Redondo, Cristina, Normas y actitudes normativas, México, Distribuciones Fontamara, 1994 (Biblioteca de Ética, Filosofía del Derecho y Política, Núm. 36), p. 9.

${ }^{16}$ ¿No es acaso el dictar órdenes y establecer reglas la manifestación natural de la autoridad (jurídica)? Para usar las propias palabras de Joseph Raz (Cf.: Practical Reason and Norms, cit., p. 21). 
cepción de normas, a saber: que $N$ es aplicada (impuesta) a los súbditos (de aquí en adelante b). Cuando se dice que " $N$ se aplica a" o que " $N$ se impone a" equivale a decir " $N$ prescribe". Más aún, y la mayoría de los juristas (tanto de la tradición romano-germánica como de la tradición del Common Law) como de los teóricos del derecho, admiten que " $N$ prescribe" significa: "la conducta de algún individuo (la conducta de un súbdito) se encuentra ordenada, prohibida o (expresamente) permitida".

Ese es el «punto de encuentro» de las teorías jurídicas.

Ciertamente, cuando hablo de normas me refiero a normas positivas que pertenecen a órdenes jurídicos que existen. ${ }^{17}$ Así, tenemos:

$$
D=\{N, N, N, N \ldots\}
$$

Donde $N$ es cualquier cosa que se hace norma. $\mathbf{x}$ se hace $\boldsymbol{N}$ si ciertas condiciones son satisfechas. Así:

$$
D \subset m
$$

d. Epílogo: Dos clases de cosas

De lo anteriormente dicho se sigue que existen dos diferentes criterios de identidad para dos diferentes clases de cosas.

Una:

$\boldsymbol{x}$ se convierte en $\boldsymbol{R}$ si, y sólo si, (cualquier hombre racional) considera a $\boldsymbol{x}$ como razón (prima facie) para actuar o para abstenerse de actuar. En otras palabras: $\boldsymbol{x}$ se convierte en $\boldsymbol{R}$ si, y sólo si, $\boldsymbol{A}$ considera que $\boldsymbol{x}$ es una razón prima facie para realizar $\boldsymbol{\emptyset}$ o una razón prima facie para abstenerse de $\boldsymbol{\sigma}^{18}$

Dos:

$\boldsymbol{N}$ es $\boldsymbol{N}$ si, y sólo si, ha sido establecida por $\mathcal{L}$ como tal. ${ }^{19}$

\section{La falacia}

El open question argument de Moore nos impide definir normas (jurídicas) en términos de razones, como si ser una razón fuera una «cualidad natural» de las normas. Si en cualquier definición propuesta, "norma" significa "razón”, entonces cabe preguntar: ¿Es una norma una razón? $?^{20}$

\footnotetext{
${ }^{17} \mathrm{U}$ órdenes jurídicos que han existido.

${ }^{18}$ Siendo $ø$ una acción (efectiva o potencial) de A.

${ }^{19} \mathrm{Y}$ es eficaz.
}

${ }^{20}$ Siguiendo el argumento de Moore, mi tesis es que si la cuestión tiene sentido (esto es, si la respuesta negativa no es completamente contradictoria), entonces la definición no puede ser correcta, puesto que una definición, se supone, tiene que preservar el significado del término definido. Y si lo hace, la pregunta del tipo que hice sería absurda para cualquiera que entienda el significado de la palabra "norma". (Vid.: Hepburn, R.W. "The Naturalistic Fallacy», en Honderich, Ted (Ed.), The Oxford Companion to Philosophy, Oxford University Press, 1995, p. 606);... «Moore and the Naturalistic Fallacy», en 20th Century Western Ethics, Encyclopaedia Britannica, CD, Encyclopaedia Britannica, Inc. 1995. 
¿No acaso esta última pregunta causa molestia, incomoda, por decir lo menos?

El hecho de que pueda describir normas en términos de razones no hace que las normas pierdan su carácter de normas. Ciertamente, puedo describir normas como motivos, causas, estímulos, et sit cetera; pero las normas son normas en virtud de que satisfacen la regla de reconocimiento de normas, con independencia de si satisfacen o no otro criterio en virtud del cual son reconocidas como motivos, causas, estímulos o razones. Esto trae como resultado una rapsodia de diferentes clases de cosas. Esto nos obliga a desenredar este lío.

Primero que nada preguntaría: ¿es verdad que una norma es una razón (o un tipo de razón) sólo porque un $\mathcal{L}$ legislador la ha establecido? Este enunciado, como ya hice notar, es falso. Pero si este enunciado fuera verdad los enunciados (9) " $D$ es un conjunto de $\boldsymbol{N}_{(S)}$ " y (10) " $\boldsymbol{N} \in=\mathbb{D}$ " podrían ser naturalmente sustituidos por (11) " $\boldsymbol{D}$ es un conjunto de $\boldsymbol{R}_{(S)}$ " y (12) “R $=\mathbb{D}$ ". Creo fuertemente que estos últimos enunciados son del todo extraños en cualquier discurso jurídico (y, también, en el discurso ordinario).

¿Son tan cándidos los juristas que les tomó prácticamente un milenio percatarse de que las normas no eran sino razones? Un orden jurídico como conjunto de razones que funcionen como razones para todos los agentes es impensable. Es realmente difícil concebir que "norma" pueda ser equivalente a "razón". ${ }^{22}$ Bastaría con preguntar nuevamente si es verdad que cualquier $N$ establecida por el legislador funciona como una razón para todos en toda circunstancia.

\section{La objeción de ignorantia iuris}

Ignorantia iuris neminen excusat, ${ }^{23}$ reza la máxima. La ignorancia del derecho, en general, no excusa su cumplimiento. A nadie se le permite excusarse sobre la base de la ignorancia del derecho. ${ }^{24}$ Es ampliamente sabido que las normas (jurídicas) funcionan de esta manera (i.e. como normas), aun cuando la gente no las conozca o no reconozca que una norma sea una norma. Como señalé anteriormente, las razones son razones porque $\mathbb{d}_{(s)}$ (los agentes) que actúan sobre la base de ellas las tratan como tales. $\mathbb{d}_{(s)}$ (la

${ }^{22}$ Tenemos que recordar que (el agente) es el hombre que actúa sobre la base de razones que reconoce como tales, y $\$$ (el súbdito) es el hombre a quien se le aplica el derecho.

${ }^{23}$ Vid.: Adolf, Berger, Encyclopaedic Dictionary of Roman Law, Philadelphia, The American Philosophical Society, 1968, p. 491. Para conocer algunos lineamientos de la doctrina, véase: D. 22, 6, 1-6. Los bizantinos firmemente sostenían: "Constitutiones principum [léase: ius] nec ignorare quemquam nec dissimulare permittimus.» (C. 1, 18, 12). 1980, p. 599.

${ }^{24}$ Vid.: Walker, David M., Oxford Companion to Law, Oxford, Oxford University Press, 
gente) adopta (por así decirlo) razones. De otra manera no podría haber razones (para sus acciones). Tenemos razones que adoptamos, sin embargo las normas no tienen que ser adoptadas, las normas simplemente se aplican a la gente. El derecho se aplica ex auctoritas y la ignorancia de sus normas no excusa a nadie. ${ }^{25}$ (el agente) no es \& (súbdito). ¿No es este hecho particular un rasgo característico de la autoridad (o fuerza) de las normas? Las normas son normas con independencia de las consideraciones de los súbditos. ${ }^{26}$

¿No es esto una diferencia definitiva? ¿qué no es claro que las normas y las razones operan de forma distinta? ¿qué son diferentes?

\section{La «tijera de Bentham»}

La «navaja de Ockham» ${ }^{27}$ podría ser aplicada aquí para desenredar la confusión entre razones y normas. Si las normas son normas y las razones, razones, tenemos que tratarlas (explicarlas) de forma separada.

De hecho, Bentham aplica estrictamente el principio de Ockham en su clara distinción entre derecho y moral, ${ }^{28}$ señalando simplemente que si realmente podemos describir el derecho sin recurrir a argumentos morales (como realmente hacemos), entonces tenemos que tratar al derecho y a la moral por separado.

De la misma manera: normas y razones. Realmente uno puede describir una norma (y los hechos sociales que la establecen) sin recurrir al concepto de razón.

${ }^{25} \mathrm{Si}$ algunas excusas son admitidas es el propio derecho que las permite. De hecho, la doctrina de la ignorantia iuris está construida para justificar exceptiones.

${ }^{26}$ Ciertamente tengo presente los problemas de la consuetudo contra legem y del tacitus consensus populi (incluyendo la legitimidad), sin embargo voy a ignorar estas cuestiones en el presente ensayo.

${ }^{27}$ El celebrado principio: non sunt multiplicanda entia praeter necessitatem (i.e., las entidades no deben ser multiplicadas más haya de lo necesario), también llamado "Ley de la economía" o "Ley de la parsimonia", fue establecido por Guillermo de Ockham (1285-1347/49). Ockham mencionaba su principio con frecuencia y lo usaba tan «filosamente» (so sharply) que fue llamado "la navaja de Ockham" (Vid.: Encyclopaedia Britannica, CD-ROM, Enciclopaedia Britannica Inc. 1995).

${ }^{28}$ En cuanto a la expresión "tijera de Bentham"» fue, en principio, un error; quise decir: "la navaja de Bentham" en alusión directa a la «navaja de Ockham», pero, tontamente, escribí "tijera" (supongo porque ambos utensilios cortan). Imperceptiblemente ese error lo repetí varias veces en clase. El hecho es que, incluso, ha aparecido publicado. (Vid.: mi ensayo «Dogmática jurídica y teoría moral», Isonomía, Revista de Teoría y Filosofía del Derecho, Instituto Tecnológico Autónomo de México, Abril 1996, Núm. 4, pp. 32-34) y mi libro: Elementos para una teoría general del derecho, México, Themis, 1998, p. 401) Algunos colegas (a quienes seguramente les hace gracia la expresión) me han pedido que lo conserve. 
Es prácticamente obvio que los abogados y los juristas pueden determinar la existencia de las normas (y sus contenidos) sin necesidad de hablar de razones (para la acción).

Si tales enunciados son verdaderos como pienso que lo son, de ahí se sigue que las normas no pueden ser correctamente definidas como razones para la acción aun cuando las normas puedan ser consideradas una razón para la acción al describir el comportamiento del agente (sus intereses o deseos). De esta manera, Bentham diría (¿diría?) que el criterio de existencia de una norma es completamente diferente del criterio de existencia de una razón (para la acción). La existencia de una norma vis à vis de los súbditos es algo que simplemente pasa. Esto es, si conocemos lo que una norma es y cómo opera en el orden jurídico sin recurrir al concepto de razón, entonces las normas (desde un punto de vista jurídico) no pueden ser definidas en términos de razones para la acción. ${ }^{29}$

No obstante, es verdad que cuando $\mathcal{L}$ (el legislador) establece una norma, habitualmente pretende que esa norma se convierta en una razón para que los súbditos actúen (en consecuencia).

Pero el acto de voluntad de $\mathscr{L}$ no es una condición suficiente para que $\boldsymbol{N}$ sea $\boldsymbol{R}$ para que todo $b$ actúe. La relación «norma-destinatario» no es en ningún sentido similar a la relación «razón-agente»: las razones dependen de las consideraciones del agente, las normas ignoran las consideraciones del súbdito.

\section{Normas como razones de segundo orden}

\section{Revisión}

Las normas, como ya señalé, pueden ser tomadas como razones para la acción. Un agente puede actuar sobre la base de una norma, tomándola como razón para su acción (u omisión). actúa sobre la base de una norma si ésta es una razón que vence otras razones. Si Actúa sobre la base de la norma $N$, esto sólo significa que esta norma particular, habiendo sido tomada en consideración por $\boldsymbol{A}$, es, además, una razón $(\boldsymbol{R})$ para que él actúe. En todo caso será una razón victoriosa, una razón que supera otras razones de $\mathbb{A}^{30}$ Por el contrario, si no actúa sobre la base de la norma que

${ }^{29}$ Jeremías Bentham encuentra que el derecho se presenta bajo una máscara y mucho de lo que escribió está destinado a quitársela. (Cf. Hart, H.L.A., Essays on Bentham. Jurisprudence and Political Theory, Oxford, Oxford University Press, 1982, p. 2). No debemos ponérsela de nuevo.

${ }^{30} \mathrm{Si}$ actúo sobre la base de una norma puedo decir que «obedezco la norma». Sin embargo, esto no significa que siempre que me conforme con una norma equivalga a obedecerla. Conformarse con una norma puede depender de otras razones. Puede suceder que yo ignore la norma y, sin embargo, la cumplo si mi acto se conforma con lo que ella establece. Dicho bre- 
ha tomado en cuenta, esto simplemente significa que $\boldsymbol{N}$ ha sido superada por otras razones de más peso que el agente pudo haber tenido (por ejemplo: una ideología particular, una decisión de desobediencia civil o, incluso, una razón trivial).

Las normas, como señalé anteriormente, pueden ser tomadas como razones (como cualquier cosa) si satisfacen lo que he denominado "la regla de reconocimiento de razones", esto es, si son reconocidas (consideradas) como tales por (el agente). Ésta es la única forma de que las normas entren dentro de la clase de cosas a las cuales el agente les asigna cierta relevancia: la clase de «el balance de razones del agente». ${ }^{31}$

La resistencia a esta idea es, sin embargo, muy fuerte. Muchos autores piensan que la autoridad de las normas jurídicas depende del hecho de que las normas son razones de cierto tipo.

\section{La noción}

En lo que toca a este concepto me mantendré dentro de los límites de la teoría de Joseph Raz. Este conocido estudioso de Oxford explica que las razones pueden ocupar dos diferentes niveles: pueden ser razones ordinarias o bien razones de segundo orden. Las razones ordinarias son razones para la acción o para abstenerse de la acción. Esta idea puede ser claramente expresada por "R $\boldsymbol{\emptyset}$ ” y por "R $\sim \boldsymbol{\emptyset}$ ” (donde " $\boldsymbol{R}$ ”es una razón para realizar ø, "ø" es una acción específica y “ ø" su omisión). Por el contrario, las razones de segundo orden no son razones para la acción inmediata, sino para seleccionar o excluir razones para la acción. ${ }^{32}$ Esta idea puede ser expresada por " $\boldsymbol{R}_{2} \boldsymbol{r}$ " y " $\boldsymbol{R}_{\mathbf{2}} \sim \boldsymbol{r}$ " (donde " $\boldsymbol{R}_{2}$ " es una razón de segundo orden; " $\boldsymbol{r}$ " es una clase de razones y “ $\sim \boldsymbol{r}$ " su exclusión).

Raz explica en varios ejemplos la forma en que funciona $\boldsymbol{R}_{2}$. Permítaseme comenzar con el caso de las instrucciones del padre. Un padre le dice a su hijo: «obedece a tu madre». Esta instrucción es una razón para actuar por una razón: una razón para actuar sobre la base de las instrucciones de su madre, las cuales son también razones. Este estado de cosas puede ser descrito perfectamente bien por $\boldsymbol{R}_{\boldsymbol{2}} \boldsymbol{r}$, donde " $\boldsymbol{R}_{\boldsymbol{2}}$ " representa la instrucción del padre y " $\boldsymbol{r}$ " una clase de razones (las instrucciones de la madre). Raz llama a esta razón $\boldsymbol{R}_{2}$ : "razón de segundo orden positiva".

vemente: puedo ignorar las normas que se me aplican y ser considerado un hombre cumplido (un law-abiding man).

${ }^{31}$ Cf.: Practical Reason and Norms, cit., p. 36. (Razón práctica y derecho, cit., p. 40); Vid.: Gans, Chaim, «Mandatory Rules and Exclusionary Reasons», en Philosophy, Vol. 15, 1986, p. 374.

32 «...cualquier razón para actuar por una razón o para abstenerse de actuar por una razón» (Raz, Joseph, Practical Reason and Norms, cit., p. 39, [Razón práctica y derecho, cit., p. 44]). 
Según Joseph Raz, hay también razones de segundo orden negativas. Supóngase que el padre, por el contrario, le dice a su hijo: «no obedezcas a tu madre». En este caso, el hijo tiene una razón para no actuar por una razón. Esta situación puede ser descrita por " $\boldsymbol{R}_{\mathbf{2}}$ $\sim \boldsymbol{r}$ ", donde " $\boldsymbol{R}_{\boldsymbol{2}}$ " es la instrucción del padre, " $\sim \boldsymbol{r}$ " es una clase de razones (las de la madre) las cuales están excluidas. Raz llama a las razones de segundo orden negativas: "razones excluyentes". ${ }^{33}$

Pienso que la idea de las razones $\boldsymbol{R}_{2}$ es claramente captada en el ejemplo dado por Raz. ${ }^{34} \mathrm{Al}$ menos, uno puede distinguir un tipo de razones que funcionan seleccionando razones $\mathrm{y}$, ciertamente, puedo representar gráficamente $\boldsymbol{R}_{2}$ (las razones del padre) y distinguirlas de $\boldsymbol{R}_{\boldsymbol{I}}$ (las razones de la madre). En el ejemplo es igualmente fácil apreciar dos diferentes tipos de razones de segundo orden: las razones de segundo orden positivas y las excluyentes.

\section{Razones excluyentes}

\section{Primer acercamiento}

En lo que a la explicación de las normas se refiere, la noción de razón excluyente es, según esta concepción, más importante. De hecho, Raz encuentra (o encontró) en esta noción el concepto maestro para caracterizar a las normas (i.e. directivas «autoritativas» ${ }^{35}$ ).

Voy a detenerme brevemente en esta subclase de razones de segundo orden (i.e. $\boldsymbol{R}_{\mathbf{2}}$

$\sim r$ ). Si funcionan como razones excluyentes, requieren que el agente ignore (i.e. pase por alto) alguna clase o algunas clases de razones. ${ }^{36}$

Permítaseme recapitular la descripción de una razón excluyente en el caso de la instrucción del padre. Un padre ordena a su hijo no obedecer a su madre $\left(\boldsymbol{R}_{\boldsymbol{2}} \sim \boldsymbol{r}\right)$. Ésta es una razón de segundo orden que requiere que el hijo pase por alto las instrucciones de su madre (la clase de actos $\boldsymbol{r}$ ). $\boldsymbol{R}_{2}$ es clara-

${ }^{33}$ The Authority of Law. Essays on Law and Morality, Oxford, Oxford University Press, 1979, pp. 16-17 (existe versión castellana de Rolando Tamayo y Salmorán: La autoridad del derecho. Ensayos sobre derecho y moral, México, UNAM, Instituto de Investigaciones Jurídicas, 1982, pp. 31-32).

${ }^{34}$ En este ensayo no abordaré la noción de $\boldsymbol{R}_{2}$. Más bien asumo que funciona en el sentido de que hay algunos hechos (dichos, instrucciones, peticiones) que seleccionan o excluyen razones para la acción.

${ }^{35}$ Sit venia verba. "Autoritativa" resulta casi un barbarismo que debe leerse como "dotadas (las directivas) de autoridad" o simplemente "válidas".

${ }^{36}$ Como más adelante mostraré las normas requieren normalmente que el agente ignore todas las razones salvo una. El propósito de introducir razones excluyentes no consiste en modificar el balance de razones, sino excluir la acción (así ordenada) del balance de razones. En este hecho reside, según Raz, la diferencia entre órdenes (léase "normas")y peticiones ( $C f:$ Raz, Joseph. The Authority of Law, cit. p. 23. [La autoridad del derecho, cit., pp. 38-391]). 
mente diferente de $\boldsymbol{R}_{\boldsymbol{l}}$, (cualquier razón de primer orden, incluyendo cualquier miembro de $\boldsymbol{r}$, las cuales están excluidas). Asumiendo, obviamente, que la orden del padre ha sido considerada como una razón por Juan (el agente, en el caso el hijo) y, en particular, como una razón excluyente (i.e. $\left.R_{2} \sim r\right)$.

\section{La prueba del recalcitrante}

Sin duda puedo adoptar (considerar) razones de segundo orden (incluyendo razones excluyentes). Lo puedo hacer siempre que satisfagan la regla de reconocimiento de razones. Pero, además de esto, una condición adicional tiene que ser satisfecha cuando adopto (acepto) razones excluyentes: tengo que aceptarlas como tales (i.e. como razones excluyentes) y consecuentemente tengo que ignorar todas las razones que éstas excluyen. Tenemos que tener muy claro que esto sucede ${ }^{37}$ en razón de lo que hago, esto es, por el hecho de que yo adopto $\boldsymbol{N}$ como un tipo de $\boldsymbol{R}_{2} \sim \boldsymbol{r}$, y no porque ser $\boldsymbol{R}_{2}$ pudiera ser una cualidad de las normas.

Veamos una versión un tanto diferente de este ejemplo (el caso del tazón de «avena»). La instrucción (orden o directiva) es clara: "Juan, actúa sobre la base de lo que tu madre dice y no tomes en cuenta cualquier razón que pudieras tener". El espectro de posibilidades es el siguiente: (1) Juan puede adoptar la instrucción de su padre (reconociéndola como razón) y puede considerarla, además, como excluyente y, por tanto, ignorar toda razón que pudiera tener. (2) Juan puede considerar la directiva de su padre, pero sólo como una razón ordinaria. (3) Juan puede ignorar totalmente tal directiva. ${ }^{38}$

Durante un cierto tiempo Juan considera la orden de su padre como una razón excluyente y siempre que su madre le ordena algo él actúa sobre la base de lo que ella dice (incluyendo la puesta del abrigo viejo y feo ${ }^{39}$ ), ignorando cualquier razón que él pudiera tener. Pero, un buen día, la madre de Juan le dice no sólo que se ponga el abrigo viejo y feo, sino que coma un tazón de avena. Ésta fue la gota que derramó el vaso. Juan decide desobedecer sistemáticamente. A partir de entonces, Juan actúa sobre la base de sus propias razones; actúa sobre la base de sus propias razones para no comer avena: la avena se ve horrible, huele horrible y sabe horrible; su ingestión lo hace vomitar, siempre se pone malo y recordar la avena lo deprime profundamente.

Desde que Juan empezó a actuar sobre la base de sus propias razones, la directiva de su padre, eo ipso, perdió su carácter de razón excluyente. Des-

\section{${ }^{37}$ Sucede porque yo hago que suceda.}

${ }^{38}$ Juan pasa por alto esta directiva porque no le importa o porque ya aceptó una razón excluyente que le ordena actuar sobre la base de lo que su padre diga.

${ }^{39}$ Así es el ejemplo de Joseph Raz. (Cf.: The Authority of Law, cit., p. 17 [La autoridad del derecho, cit., p. 331]). 
de ese preciso momento la directiva de su padre, si no ignorada, descendió al nivel de razón ordinaria (por lo menos en lo que a la avena se refiere). No fue fácil para Juan desobedecer a su madre adorable. Fue peor desobedecer a su estricto padre (Juan sabe muy bien las consecuencias que esta desobediencia puede acarrear). Pero desde ese día, Juan no comió, no come y no comerá jamás avena, sin importar lo que pueda pasarle. De esta manera, Juan se convirtió en un recalcitrante (gracias a la avena).

Según la teoría que aquí es analizada, las órdenes (léase "directivas" o "normas") son razones excluyentes. Pero, como hemos visto, una vez que las directivas pierden su carácter excluyente, pierden también ese rasgo peculiar en el que reside su particular característica autoritativa o normativa. Si una razón no es una razón excluyente, de acuerdo con la teoría aquí analizada, no es una orden (no es una norma). Pero ¿es así? Supongamos que le preguntamos a Juan sobre la orden de su padre. ¿Cree usted que Juan piensa que la orden de su padre ha desaparecido? ¿que ya no existe? Por supuesto que no, la orden existe, es precisamente la orden que Juan desobedece. Juan podría estar pagando las consecuencias de su desobediencia.

Supongamos que las consecuencias para Juan son desastrosas y su hermano, Alberto, astutamente decide, por razones prudenciales, no desafiar a su inflexible y victoriano padre, por lo menos mientras no pueda evitar el castigo. Para Alberto, la directiva de su padre (dirigida a él también) no es adoptada (considerada) como una razón excluyente. Es una razón ordinaria. Ciertamente es una razón de peso que supera todas las razones que Alberto puede tener. Pero, cabe preguntar: ¿es o no la directiva del padre una orden para Alberto? ¿existe o no? Por supuesto es una orden, una orden que efectivamente existe, no obstante no sea una razón excluyente. Pregúntese a Alberto si no es sobre la base de la orden de su padre que él está actuando, aunque ésta no sea una razón excluyente.

Es realmente paradójico que el rasgo característico de las órdenes (normas o directivas) pueda ser eliminado de ciertos hechos y que éstos continúen comportándose como órdenes (normas). Esto debe recordarnos la objeción de ignorantia iuris o la tijera de Bentham: las normas son normas aun cuando no sean consideradas razones excluyentes o si no son consideradas como razones en absoluto.

Lo que pasa con Juan y su padre (y con Alberto), pasa igual con una norma jurídica, por ejemplo, con una resolución de un juez. Simplemente supóngase que la instrucción del padre es una decisión judicial que ordena que la parte convicta pague una suma de dinero (y que ignore, $a b$ obvo, cualquier otra razón que pudiera tener). El espectro de las posibilidades de la parte perdedora es exactamente igual al que enfrenta Juan, a saber: (1) Puede adoptar (considerar) la decisión del juez y tomarla como una razón ex- 
cluyente y, por tanto, ignorar las otras razones que pueda tener. (2) Puede considerar la decisión del juez, pero sólo como razón ordinaria. El convicto es un capo y como pantomima decide, por razones prudenciales, someterse al derecho. (3) Puede ignorar por completo esta directiva (es recalcitrante como lo fue Juan).

En el extremo, la gente puede nunca reconocer que una directiva es una razón excluyente. La gente puede ignorar las normas no obstante se sostenga que obedecer normas es un tipo de racionalidad. De hecho, la idea de describir normas (directivas u órdenes) como razones excluyentes está encubierta con sutiles argumentos a través de los cuales se introduce la idea de racionalidad o de autoridad que, de alguna manera, se encuentran detrás de las normas jurídicas.

Pasemos a otro ejemplo. Imaginémonos un pasajero (en el caso del naufragio ${ }^{40}$ ) que defiende tenazmente su autonomía moral y en nombre de la sacrosanta racionalidad decide ponderar (reconsiderar) todas las órdenes dadas por el capitán. Este hombre (posiblemente el único pasajero ahogado) no reconoce las órdenes del capitán como razones de segundo orden, sino sólo como razones ordinarias. Aquí tenemos el vívido (o fatal) ejemplo de un agente que rehúsa pasar por alto otras razones (ordinarias). En razón de su testarudez, las órdenes del capitán no cuentan lo suficiente en su balance de razones. Pero supongamos qué no obstante su necedad, miembros de la tripulación lo hacen obedecer. En este caso las órdenes del capitán son normas, normas que se aplican incluso a los recalcitrantes, contra su voluntad (y contra su balance autónomo de razones).

\section{Segunda aproximación}

Joseph Raz proporciona otros ejemplos de razones de segundo orden, los cuales, de alguna manera, contrastan con los que he analizado anteriormente. Todos muy claros. Sin duda. En ese aspecto no hay problema. El problema radica en la forma en que operan en ellos las razones excluyentes. Haré un pequeño resumen de los ejemplos que proporciona Raz, los casos: Ana, Jeremías y Colín. ${ }^{41}$

Ana. Ana está muy cansada y, en tal virtud, por su fatiga, decide no considerar una complicada oferta de inversión y, consecuentemente, rechaza la oferta. ${ }^{42}$

${ }^{40}$ Raz toma este ejemplo de R.P. Wolff ( $C f$.: en Defense of Anarchism, Nueva York, Harper and Raw, 1970, p. 14). Raz usa este ejemplo para mostrar que la aceptación de la autoridad requiere de la existencia de «razones de segundo orden válidas» (Cf.: The Authority of Law, cit. pp. 26-27 [La autoridad del derecho, cit., pp. 41-43]).

${ }^{41}$ Vid.: Gans, Chaim. «Mandatory Rules and Exclusionary Reasons», cit., p. 374.

${ }^{42}$ Cf.: Practical Reason and Norms, cit., p. 39 (Razón práctica y normas, cit., pp. 42-43). 
Jeremías. Jeremías es un soldado. Su superior le ordena tomar un vehículo que pertenece a un civil. Jeremías, en el caso que analizamos, está consciente del hecho de que tomar el vehículo es un exceso y conoce mejores formas para lograr lo que su superior quiere. Jeremías es un buen soldado y, no obstante sus propias consideraciones, recuerda que «...órdenes son órdenes y deben ser obedecidas aun sean equivocadas. ...»» ${ }^{43}$

Colín. Colín promete a su esposa que en todas las decisiones que afecten a su hijo él actuará únicamente viendo el interés de su hijo, ignorando cualquier otra razón. Colín tiene fuertes razones para actuar de tal manera que no corresponde completamente con lo prometido, sin embargo cumple con su promesa y pasa por alto las fuertes razones que tenía. $^{44}$

La noción de razón excluyente es claramente descrita en los ejemplos dados por Raz. Sin duda podemos distinguir cierto tipo de actos (la fatiga, una orden, una promesa) que funcionan excluyendo razones: todas las razones que pertenecen a la clase de miembros del balance de razones excepto ellas mismas.

Estos tres casos difieren de aquél de la instrucción del padre. En este último existen dos instrucciones diferentes. La primera, la del padre (una razón para no actuar por una razón, i.e. $\left.\boldsymbol{R}_{2} \sim \boldsymbol{r}\right)$ y, la segunda, la de la madre. Consecuentemente, tenemos dos tipos de instrucciones, emitidas por dos diferentes fuentes, en dos diferentes momentos. Si ambas instrucciones (consistentes en dos hechos completamente diferentes) satisfacen la regla de reconocimiento de razones, ambas funcionan como razones para el agente. En los otros tres casos, no hay dos actos de emisión de instrucciones (por así decirlo). Raz considera la fatiga de Ana, la orden del superior y la promesa de Colín como una sola y única razón que el agente tiene que enfrentar (una razón, de alguna manera, externa a aquellas que pertenecen al balance de razones que tienen que ser pasadas por alto o excluidas). Joseph Raz señala que «...el mismo hecho [como sucede en los tres últimos casos] es, al mismo tiempo, una razón para la acción y una razón (excluyente) para pasar por alto las razones en su contra» ${ }^{45}$. De esta peculiar opinión resulta un tipo de enredo.

\section{El enredo}

Voy a analizar el caso de Jeremías en donde una norma es dictada. Dos diferentes aspectos entran en juego aquí. El primero, la orden dictada, y el segundo, el hecho de que uno tiene que pasar por alto todas las otras ra-

\footnotetext{
${ }^{43}$ Cf.: Practical Reason and Norms, cit., p. 38 (Razón práctica y normas, cit., p. 43).

${ }^{44}$ Cf.: Practical Reason and Norms, cit., p. 39.

${ }^{45}$ The Authority of Law, cit. p. 18. (La autoridad del derecho, cit., p. 33).
} 
zones (de primer orden). Lo que es difícil de aceptar es la tesis de Raz de que estos dos aspectos se encuentran en la sola y misma orden «toma el vehículo» dada por el superior.

En el caso de Jeremías (como en los otros casos) no es problema separar los dos niveles de consideraciones. Uno: la orden dada por el superior «toma el vehículo» (una razón de primer orden) y dos, otra proposición: ${ }^{46}$ "las órdenes (militares) deben ser obedecidas sin considerar sus propios méritos" (una razón de segundo orden). Esta última razón, sin duda es parte de la situación. Si no fuera así, ¿de dónde hubiera sacado Jeremías la idea de que «las órdenes son órdenes»? (para usar las mismas palabras de Raz).

La orden del superior $\left(\boldsymbol{R}_{1}\right)$ podría haber sido fácilmente superada si Jeremías, no siendo soldado, hubiera podido considerar y ponderar otras razones para decidir. Estas razones no fueron siquiera consideradas porque Jeremías, previamente, adoptó otra directiva: "las órdenes (militares) deben ser obedecidas sin considerar sus propios méritos", en otras palabras: "órdenes son órdenes", es una razón $\boldsymbol{R}_{2}$ que gobierna la acción de Jeremías (en lo que atañe a los asuntos militares).

De cualquier modo, $\boldsymbol{R}_{2}$ no hubiera podido ser $\boldsymbol{R}$ si Jeremías no la hubiera identificado como $\boldsymbol{R}$. El dicho "órdenes son órdenes..." en tanto que intervinieron en la consideración de Jeremías, es una razón para que él actúe. Y siendo una razón $(\boldsymbol{R})$, es, además, tomada como una razón de segundo orden, simplemente porque Jeremías decide jugar el juego del soldado. ${ }^{47}$

De hecho Jeremías enfrenta dos diferentes situaciones. La primera es la decisión de «jugar al soldado» y la segunda, es la orden del superior. ${ }^{48}$ Jeremías obedecerá las órdenes de su superior mientras continúe «jugando al soldado». Jeremías puede abandonar (él puede desertar e, incluso, resistir a la autoridad), sin embargo hasta ahora él tiene que jugar el «juego». Este «juego» es fácil de jugar. Como cualquier otro juego, este juego se juega obedeciendo, únicamente con «jugadas del juego», las órdenes militares superiores.

En contraposición con esto, Raz considera la orden del superior (en realidad una única razón) como si fuera al mismo tiempo una razón de segundo orden y una razón de primer orden.

No cabe duda que en casos como los de Jeremías existen $\boldsymbol{R}_{\boldsymbol{1}}$ y $\boldsymbol{R}_{2}$. Esto es indisputable. Es igualmente indisputable que $\boldsymbol{R}_{1}$ evoca o presupone $\boldsymbol{R}_{2} \cdot{ }^{49}$

${ }^{46}$ Presupuesta en la orden del superior. Lógicamente una proposición anterior.

${ }^{47}$ Puede ocurrir que la decisión de jugar al soldado no sea siempre completamente «libre», como sucede en los casos de reclutamiento forzoso y leva.

${ }^{48}$ Aunque estas dos diferentes situaciones son generalmente dos diferentes momentos, puede ocurrir que estos dos momentos puedan ocurrir en acciones del agente que se presenten prácticamente de forma simultánea. Sin embargo es fácil observar que la decisión de jugar al soldado y la orden del superior son dos hechos totalmente diferentes.

${ }^{49} \mathrm{La}$ orden del superior, de alguna manera, presupone la regla fundamental del juego. 
Sin embargo, el hecho de que una razón evoque otra razón no es suficiente para tratarlas como si fueran una y la misma razón. La construcción de esta «razón excluyente-de primer orden» es realmente una entidad extraña.

Esta rara razón (de segundo orden-excluyente-de primer orden) es la clave de la explicación de las normas. De hecho según Raz este dato constituye el rasgo característico de las normas. Así, de conformidad con esta tesis, $\boldsymbol{N}$ es una entidad que participa de dos características incompatibles: es una razón de primer orden y una razón de segundo orden excluyente, al mismo tiempo, una criatura hipostática, realmente mitológica. ¿Cómo puede ser que un mismo hecho (i.e. una directiva) pueda ser, para el mismo agente y en una misma situación, una $\boldsymbol{R}_{1}$ y una $\boldsymbol{R}_{2}$ a la vez $?^{50}$

\section{Argumentación (razonamiento) jurídico}

\section{El planteamiento}

¿Cuándo, por qué y para qué usamos normas como razones (para la acción)? Una respuesta es la siguiente: usamos normas para respaldar (justificar) jurídicamente o desacreditar la conducta humana, esto es lo que la profesión jurídica ha hecho por milenios. En este momento quiero distinguir dos cuestiones que reflejan dos diferentes situaciones: la situación de primer nivel (en la cual el agente no juega juego alguno) y el punto de vista jurídico (en el cual se presenta una regla de segundo orden que identifica las «jugadas del juego»). En cuanto a la situación de primer nivel, la hemos abordado en los casos analizados anteriormente. En cuanto a la situación del punto de vista jurídico, éste se parece notablemente a la situación en que encontramos a Jeremías frente a las órdenes de su superior.

Voy a sostener la tesis de que la argumentación y, en particular, la argumentación jurídica, puede ser descrita en cercana analogía con jugar juegos. Al menos con el de Jeremías, cuando decide jugar al soldado.

Al principio de este ensayo señalé que dos preguntas habían afectado profundamente a la humanidad, a saber: “¿qué es?” y “qqué hacer?”. Consecuentemente podemos distinguir dos problemas: ¿qué es una norma?, por un lado, y ¿qué hacer con las normas?, por el otro. Así, resulta muy importante distinguir entre la descripción de (la naturaleza) de una norma jurídica y la forma como ésta puede funcionar como una razón (si así es considerada).

\footnotetext{
${ }^{50}$ Una orden realmente primera, dada realmente por el primer líder sobre la tierra, aunque sea una y misma orden, presupone una norma fundamental: "obedece al primer líder (y pasa por alto otras razones)". Ésta es la única forma de que alguien sea primer líder. Si algunos hombres le obedecen es porque han adoptado la norma fundamental: una norma que no fue establecida por el primer líder.
} 
En este mismo orden de ideas, es importante distinguir entre la aplicación de normas $^{51}$ y la argumentación jurídica (i.e. el juego de la justificación jurídica), juego básicamente gobernado por la dogmática jurídica.

En cuanto a la cuestión "¿qué hacer con las normas (jurídicas)?” observo las situaciones siguientes:

O bien:

(10) Puedo ignorar $\boldsymbol{N}$ (ningún juego se juega)

(11) puedo tomar en cuenta (considerar) $\boldsymbol{N}$.

En este último caso, surgen tres situaciones posibles:

(11.1) Puedo actuar sobre la base de $N$, si $\boldsymbol{N}$ vence a las razones en contra ${ }^{52}$ (ningún juego se juega),

O bien:

(11.2) Puedo actuar de otra manera no obstante $N^{53}$ (ningún juego se juega)

(11.3) Considero $\boldsymbol{N}$ y decido que $\boldsymbol{N}$ sea el único tipo de razones sobre la base de las cuales voy a actuar (un juego se juega).

En este último enunciado se encuentra la clave para entender el juego de la argumentación jurídica.

\section{Juguemos (juegos)}

Supongamos que el padre de los anteriores ejemplos decide jugar ajedrez con Juan, su hijo. El padre, irritado por el curso que ha tomado el juego, decide mover su «rey» cinco cuadros de manera diagonal, arguyendo que si los «alfiles» lo pueden hacer, el «rey» no puede ser menos. No obstante el argumento, Juan descalifica la acción diciendo que no es una jugada del juego. Más tarde, el padre de Juan objeta airadamente un «enroque» realizado por Juan, arguyendo que es injusto toda vez que él estaba atacando ciertos cuadros $\mathrm{y}$, ahora, el «enroque» hace que sus jugadas sean completamente inútiles. A esto Juan contesta que el «enroque» es una jugada del juego. Después de esto, el padre de Juan pierde un peón por una captura «al paso». El padre, alegando que ignoraba que se pudiera «comer al paso» reclama la devolución de su peón. Juan dice que «comer al paso» es una jugada del juego y, por tanto, la jugada se mantiene. Manifiestamente incómodo, el padre de Juan interrumpe el juego diciendo a su hijo: «estoy harto de tu estúpido estribillo "una jugada del juego". ¡ Vete a tu cuarto! Voy a

${ }^{51}$ Cómo son aplicadas a los súbditos por los órganos jurídicos.

${ }^{52}$ Yo actúo sobre la base de $N$ si, además del hecho de que las razones en contra de la acción han sido vencidas, actuaría de otra manera si $N$ no hubiera sido tomada en cuenta en mi deliberación. para actuar.

${ }^{53} \mathrm{~N}$ es una razón vencida por otras razones o es sólo una razón complementaria (pero débil) 
continuar el juego con "jugadas" de mi propio juego. En esta casa soy yo quien decide qué es una "jugada del juego"». Sin embargo, el padre de Juan sabe perfectamente bien que esto no es verdad y que si quiere ganarle a su hijo un juego de ajedrez, la única manera de hacerlo es usando únicamente «jugadas del juego» y no con autoridad paterna.

Juan, prudentemente, se fue a su cuarto jugando el juego del «hijo obediente», sabiendo que el ajedrez, como cualquier otro juego, se juega exclusivamente con jugadas reconocidas como jugadas del juego.

La moraleja de este ejemplo es que cuando decidimos jugar juegos adoptamos la regla fundamental del juego, la cual podría formularse como sigue:

Juega sólo con jugadas del juego y pasa por alto cualquier razón que puedas tener.

Es claro que la regla fundamental del juego funciona como una razón de segundo orden que selecciona las razones que están admitidas en el juego (ninguna otra es admitida). En otras palabras: la regla fundamental del juego nos dice que pasemos por alto (que ignoremos) cualquier otra cosa que no sea una jugada del juego. En la confrontación el juego impone a los jugadores un simple código: haz jugadas del juego.

Las «jugadas del juego» son identificadas por las reglas que constituyen el juego (las reglas que lo gobiernan ${ }^{54}$ ). De esta forma, la regla fundamental del juego puede ser reformulada como sigue:

Al jugar juegos, actúa únicamente sobre la base del conjunto de reglas que identifican sus jugadas (y, $a b o b v o$, ignora cualquier otra consideración).

No es difícil entender que si decidimos $^{55}$ jugar un juego tenemos que pasar por alto cualquier razón que no pueda ser identificada como jugada del juego. Cuando jugamos juegos adoptamos la regla fundamental del juego (la grundnorm), la cual funciona como una razón de segundo orden.

\section{El «juego» del derecho}

Lo que los jugadores (quae jugadores) hacen con las reglas del ajedrez o con las reglas de cualquier otro juego, algunos individuos (en su mayoría, miembros de la profesión jurídica), lo hacen con las normas jurídicas cuan-

${ }^{54}$ Sobre este particular, Vid.: Robles, Gregorio, Las reglas del derecho y las reglas del juego, México, UNAM, Instituto de Investigaciones Jurídicas, 1988; Schauer, Frederick, Playing by the Rules: A Philosophical Examination of the Rule-Based Decision-Making in Law and in Life, Oxford, Oxford University Press, 1991.

${ }^{55}$ Repetidamente he escrito las diferentes inflexiones del verbo “decidir" en «negrillas», solo para subrayar la idea de Philippa Foot adelantada al principio de este ensayo en el sentido de que las razones para la acción tienen más que ver con las inclinaciones y las decisiones del agente. 
do deciden jugar el juego de la «argumentación jurídica» y adoptan la correspondiente norma fundamental.

Es muy importante tener en mente que una cosa es considerar a las normas desde el punto de vista de (situación de primer orden) y muy otra es jugar a «la argumentación jurídica». En este «juego» los jugadores tienen que pasar por alto todo tipo de razones salvo uno: el que constituye el punto de vista jurídico. El punto de vista jurídico es proporcionado por las normas jurídicas que gobiernan el «juego» ${ }^{56}$. No sólo agentes pueden jugar el juego. Es suficiente con adoptar el punto de vista jurídico: «juega actuando sólo sobre la base de normas jurídicas».

El juego es una confrontación entre dos partes (si son más, se dividen en dos bandos: con el actor o con el demandado). Tratan de probar que lo que ellos hacen (hicieron o harán) es jurídicamente justificado, de conformidad con las normas jurídicas que, consideran, se aplican a este hecho. Las partes defienden diferentes posiciones como, por ejemplo, la «naturaleza» del hecho o la definición que le corresponde o la determinación de las normas aplicables al hecho. ${ }^{57}$

La más clara expresión de la confrontación es la disputa en el tribunal. El litigio tiene que resolverse por un tercero (iudex, arbiter) quien se encuentra por encima de las partes (pro tribunale). El juez no sólo decide (resuelve) la disputa sino que además «conduce» el proceso, admitiendo o rechazando «jugadas». Ab obvo, las únicas «jugadas» admitidas son las que son reconocidas por las normas jurídicas aplicables al juego.

La confrontación puede ser hipotética y ser realizada por una sola persona.$^{58}$ Pero aun en este caso el juego adopta una forma de confrontación.

De esta manera, tenemos que ningún tipo de razones participa en esta confrontación salvo las normas jurídicas. Ciertamente, jugar el juego de la argumentación jurídica requiere de una norma fundamental del juego, la cual es una razón de segundo orden que, precisamente, establece que únicamente normas jurídicas son admitidas y que todas las otras razones son excluidas. ${ }^{59}$

${ }^{56} \mathrm{Y}$ las meta-reglas que gobiernan la profesión jurídica.

${ }^{57}$ Las partes no necesitan estar en posiciones diametralmente diferentes, es suficiente con defender diferentes puntos de vista con el propósito de obtener diferentes resultados (consecuencias).

${ }^{58}$ Cualquier individuo puede adoptar el punto de vista jurídico y decidir dar una calificación jurídica privada a un hecho particular (normalmente suyo) o consultar a un profesional. Un profesor de derecho puede adoptar el punto de vista jurídico para muchas situaciones hipotéticas. De este modo tenemos argumentos, privados, doctrinales y oficiales. Sólo los oficiales son obligatorios.

${ }^{59}$ Para los propósitos de este ensayo voy a ignorar los problemas de la «textura abierta». Me mantendré dentro de los límites del «standard work» de los pronunciamientos judiciales en los que el universo de las normas jurídicas es >: (igual o mayor) que el universo de casos. 
Jueces y abogados (en tanto tales) no se encuentran en una situación de primer orden. Ellos adoptan el punto de vista jurídico. Las partes, en tanto hacen lo que los abogados (y jueces) dicen, adoptan el punto de vista jurídico.

Esto, por supuesto, es una simplificación extrema del proceso. Todo el mundo sabe que el proceso jurisdiccional alcanzó un gran nivel de sofisticación desde tiempo atrás. La idea de este ensayo no era describir la alta complejidad del proceso jurisdiccional ni de los pronunciamientos judiciales, la idea es meramente probar que existe realmente una razón de segundo orden, detrás de estas instituciones, a saber: la regla fundamental de la argumentación jurídica.

Sólo resta decir que la aplicación de las normas para identificar (y justificar una acción) no es fácil. Corresponde a la dogmática jurídica decir cómo aplicar las normas jurídicas (determinando su peso, alcance, et sit cetera).

\section{Bibliografía}

AARNIO, AULIS, The Rational as Reasonable. A Treatise on Legal Justification. Amsterdam, D. Reidel Publishing Company, 1987 (Lo racional como razonable, Madrid, Centro de Estudios Constitucionales, 1991 (Colección el Derecho y la Justicia, Núm. 21)).

ALCHOURRÓN, CARLOS. E., «Logic of norms and logic of normative propositions», en Logique et Analyse, 1969, 12, 242-268;

ALCHOURRÓN, CARLOS E. y BULYGIN, EUGENIO, Análisis lógico y derecho, Madrid, Centro de Estudios Constitucionales, 1991. (Colección el Derecho y la Justicia, Núm. 24).

- «Definiciones y normas», en E. Bulygin et. al (Eds.), El lenguaje del Derecho, Buenos Aires, Abeledo-Perrot, 1983, pp. 11-42.

- «Norma jurídica», en Garzón Valdés, Ernesto y Laporta, Francisco J. (Eds.). El derecho y la justicia, Consejo Superior de Investigaciones Científicas, Madrid, Editorial Trotta, Boletín Oficial del Estado, 1996, pp. 133-147.

- Normative Systems, Springer, Viena/Nueva York, 1971.

- Sobre la existencia de las normas jurídicas, México, Distribuciones Fontamara, 1993 (Biblioteca de Ética, Filosofía del Derecho y Política, Núm. 39).

ALEXY, ROBERT, A Theory of Legal Argumentation. The Theory of Rational Discourse as Theory of Legal Justification, trad. por Ruth Adler i Neil MacCormick, Oxford, Oxford University Press, 1989 (Theorie der juristischen Argumentation: Die Theorie des rationalen Diskurses als Theorie der juristischen Begründung, Francfort, Suhrkamp Verlag, 1978).

- Derecho y razón práctica (trad. por Manuel Atienza, Pablo Larrañaga y Carlos de Santiago), México, Distribuciones Fontamara, 1993 (Biblioteca de Ética, Filosofía del Derecho y Política, Núm. 30).

- «La idea de una teoría procesal de la argumentación jurídica» (trad. por Carlos de Santiago), en Garzón Valdés, Ernesto (Com.), Derecho y filosofía, México, 
Distribuciones Fontamara, 1993. pp. 43-57. (Biblioteca de Ética, Filosofia del Derecho y Política, Núm. 18).

ATIENZA, MANUEL, «Argumentación jurídica», en Garzón Valdés, Ernesto y Laporta, Francisco J. (Eds.), El derecho y la justicia, cit., pp. 231-238.

- Las razones del derecho. Teoría de la argumentación jurídica, Madrid, Centro de Estudios Constitucionales, 1991 (Cuadernos y Debates, Núm. 31).

AUSTIN, JOHN L., How to Do Things with Words, Oxford, Oxford University Press, 1962.

BALDWIN, THOMAS. G.E. Moore, Londres, 1990.

BAYÓN MOHÍNO, J.C., La normatividad del derecho: Deberes jurídicos y razones para la acción, Madrid, Centro de Estudios Constitucionales, 1991 (Colección el Derecho y la Justicia, Núm. 26).

BENTHAM, JEREMY, On Laws in General, H. L. A. Hart (Ed.), Athlone Press, Londres, 1970.

BERGER, ADOLF, Encyclopaedic Dictionary of Roman Law, Philadelphia, The American Philosophical Society, 1968.

BIX, BRIAN, Law, Language, and Indeterminacy, Oxford, Oxford University Press, 1993.

CARCATERRA, GAETANO, La forza costitutiva delle norme, Roma, Bulzoni Editore, 1979.

FARRELL, MARTÍN D., «Obligaciones jurídicas y razones para actuar», en Revista de Ciencias Sociales, Facultad de Ciencias Jurídicas, Económicas y Sociales, Núm. 28, 1986, pp. 271-295, Valparaíso.

FOOT, PHILIPPA, Virtues and Vices and Other Essays in Moral Philosophy, Oxford, Basil Blackwell, 1978.

GANS, CHAIM, «Mandatory Rules and Exclusionary Reasons», en Philosophia, Vol. 15, 1986, pp. 373-394.

GUASTINI, RICCARDO, Le fonti del diritto e l'interpretazione, Milán, Guiffrè Editore, 1993.

- Lezioni sul linguaggio giuridico, Giappichelli Editore, Turín, 1985.

GUIBOURG, RICARDO A., Deber y saber. Apuntes epistemológicos para el análisis del derecho y la moral, México, Distribuciones Fontamara, 1997 (Biblioteca de Ética, Filosofía del Derecho y Política, Núm. 57).

- Derecho, sistema y realidad, Buenos Aires, editorial Astrea, 1986 (Filosofía y Derecho, Núm. 13).

- El fenómeno normativo. Acción, norma y sistema. La revolución informática. Niveles del Análisis jurídico. Buenos Aires, Astrea, 1987 (Filosofía y Derecho, Núm. 14).

HART, H. L. A., The Concept of Law, Clarendon, Oxford, 1961. (Existe versión castellana por Genaro Carrió: El concepto de derecho, Buenos Aires, Abeledo Perrot, 1992 (1963).

- «Commands and Authoritative Reasons», en Essays on Bentham. Studies in Jurisprudence and Political Theory, Oxford, Oxford University Press, 1982, pp. 243-268. 
- «Introduction», en Essays on Bentham. Studies in Jurisprudence and Political Theory, cit. pp. 1-20.

- «Legal Duty and Obligation», en Essays on Bentham. Studies in Jurisprudence and Political Theory, cit. pp. 127-161.

HOLLIS, MARTIN, Reasons in Action. Essays in the Philosophy of Social Science, Cambridge, Cambridge University Press, 1996.

HONDERICH, TED, Oxford Companion to Law, Oxford University Press, 1995.

KELSEN, HANS, General Theory of Norms, Trad. por Michael Hartney, Oxford, Oxford University Press, 1991.

- Reine Rechtslehre, Deuticke, Viena, 1960 (Teoría pura del derecho, trad. por Roberto J. Vernengo, México, UNAM, Instituto de Investigaciones Jurídicas, 1991 (1979)).

LAPORTA, FRANCISCO, Entre el Derecho y la moral, México, Distribuciones Fontamara, 1993 (Biblioteca de Ética, Filosofía del Derecho y Política, Núm. 26).

MACCORMICK, NEIL, Legal Reasoning and Legal Theory, Oxford, Oxford University Press, 1978.

MAZZARESE, TECLA, Forme di razionalità delle decisioni giudiziarie, Turín, G. Giappichelli Editore, 1996 (Analisi e Diritto. Serie Teorica, 25).

- «La interpretación como traducción. Esclarecimientos provenientes de una analogía común», trad. por Rolando Tamayo y Salmorán, en Isonomía. Revista de Teoría y Filosofía del Derecho, Instituto Tecnológico Autónomo de México, Octubre de 1998, pp. 73-97.

MIERS, DAVID y TWINING, WILLIAM, How to do Things with Rules, Vid. infra: Twinning, W. MOORE, GEORGE EDWARD, Principia Ethica, Cambridge, 1903.

NAVARRO, PABLO y REDONDO, CRISTINA, Normas y actitudes normativas, México, Distribuciones Fontamara, 1994 (Biblioteca de Ética, Filosofía del Derecho y Política, Núm. 36).

NINO, CARLOS S., La validez del Derecho, Buenos Aires, Astrea, 1985.

- «Razón y prescripciones» en Análisis Filosófico, Núm. 1, pp. 37-48, 1981.

OLIVECRONA, KARL, «Legal Language and Reality», en Essays in Jurisprudence in Honor of Roscoe Pound, Bobb Merril Co. Inc., Indianápolis (Lenguaje jurídico y realidad, trad. Ernesto Garzón Valdés, México, Distribuciones Fontamara, S.A. 1991.

The Imperative Element in Law [Der Imperative des Gesetzes].

ORTIZ, JAVIER, ¿Puede haber razones jurídicas autoritativas? en Isonomía. Revista de Teoría y Filosofía del Derecho, Instituto Tecnológico Autónomo de México.

- Razones para actuar sistemas jurídicos y desobediencia civil, México, Distribuciones Fontamara, (Biblioteca de Ética, Filosofía del Derecho y Política [en prensa].

PARFIT, DEREK, Reasons and Persons, Oxford, Oxford University Press, 1984. 
PERRY, STEPHEN R., «Interpretation and Methodology in Legal Theory», en Marmor, Andrei (Ed.), Law and Interpretation. Essays in Legal Theory, Oxford, Oxford University Press, 1995, pp. 97-135.

- «Second-Order Reasons, Uncertainty and Legal Theory», en Southern California Law Review, Vol. 62, 1989, pp. 913-994.

PERELMAN, CHAYM, La lógica jurídica y la nueva retórica, trad. por Luis Díez Picazo, Madrid, Civitas, 1989 (Id. Logique juridique-Nouvelle rhetorique, París, Dalloz, 1976.

RAZ, JOSEPH, «Autoridad, derecho y moral», en Isonomía. Revista de Teoría y Filosofía del Derecho, Instituto Tecnológico Autónomo de México [en prensa] («Authority, Law, and Morality» en The Monist, Vol. 68, Núm. 3, 1985).

- «Autoridad y justificación», trad. por Rolando Tamayo en Isonomía, Revista de Teoría y Filosofía del Derecho, Instituto Tecnológico Autónomo de México [en prensa]. («Authority and Justification» en Philosophy \& Public Affairs, Vol. 14, Núm. 1, 1985, pp. 3-29.

- «El problema de la naturaleza del derecho», trad. por Rolando Tamayo in Isonomía, Revista de Teoría y Filosofía del Derecho, Instituto Tecnológico Autónomo de México, («The Problem about the Nature of Law», en Gottorm, Flýstad [Ed.]. Contemporary Philosophy. Vol. 3. Philosophy of Action, The Hague, Martinus Nijhoff Publishers, 1983, también en University of Western Ontario Law Review, Vol. 21, Núm. 2. December, 1983, pp. 203-218. Después en Raz, Joseph, Ethics in the Public Domain. Essays in the Morality of Law and Politics, Oxford, Oxford University Press, 1994, pp. 179-193).

- Ethics in the Public Domain. Essays in the Morality of Law and Politics, op. ul. cit.

- «Facing Up A Reply» en Southern California Law Review, Vol. 62, 1989, pp. 1153-1235.

- «Interpretation without Retrieval», en Marmor, Andrei (Ed.), Law and Interpretation. Essays in Legal Theory, Oxford, Oxford University Press, 1995. pp. 155-175.

- «Reasons for Actions, Decisions, and Norms» en Raz, Joseph (Ed.), Practical Reasoning, Oxford, Oxford University Press, 1978, pp. 128-143.

- «¿Por qué interpretar?», en Isonomía. Revista de Teoría y Filosofía del Derecho, Instituto Tecnológico Autónomo de México, Núm. 5, Octubre 1996, pp. 25-40. México.

- Practical Reason and Norms, Hutchinson, Londres, 1975 (Razón práctica y normas, trad. Juan Ruiz Manero, Madrid, Centro de Estudios Constitucionales, 1991. [Colección el Derecho y la Justicia, Núm. 271).

- The Authority of Law. Essays on Law and Morality, Oxford, Oxford. University Press, 1979. (Existe versión castellana de Rolando Tamayo y Salmorán: La autoridad del derecho. Ensayos sobre derecho y moral, México, UNAM, Instituto de Investigaciones Jurídicas, 1982.

- The Morality of Freedom, Oxford, Oxford University Press, 1986.

REDONDO, CRISTINA, «¿Razones internas s. razones externas?», en Isonomía. Revista de Teoría y Filosofía del Derecho, Instituto Tecnológico Autónomo de México, Núm. 4, abril, 1996, pp. 135-145.

REDONDO, CRISTINA y NAVARRO, PABLO, Normas y actitudes normativas, op. cit., Vid. supra: Navarro, Pablo. 
ROBLES MORCHÓN, GREGORIO, Introducción a la teoría del derecho, Madrid, Editorial Debate, 1988.

- Las reglas del derecho y las reglas de los juegos. Ensayo de teoría analítica del derecho, México, UNAM, Instituto de Investigaciones Jurídicas, 1988, Serie G, Estudios Doctrinales, Núm. 14.

ROSS, ALF, Directives and Norms, Routledge and Kegan Paul, Londres, 1968.

SCHAUER, FREDERICK, Playing by the Rules: A Philosophical Examination of the Rule-Based Decision-Making in Law and in Life, Oxford, Oxford University Press, 1991.

SHINER, ROGER, Norm and Nature. The Movements of Legal Thought, Oxford, Oxford University Press, 1992.

TAMAYO, ROLANDO, «Dogmática jurídica y teoría moral», i.e. «Legal Dogmatic and Moral Theory» (Isonomía. Revista de Teoría y Filosofía del Derecho, Núm. 4, Instituto Tecnológico Autónomo de México, Abril 1996, pp. 32-34).

- «El sujeto del derecho», en Garzón Valdés, Ernesto y Laporta, Francisco J. (Eds.), El derecho y la justicia, op. cit., pp. 293-312.

- Elementos para una teoría general del derecho, México, Themis, 1992.

- «Lenguaje del derecho y demiurgia jurídica. Entre actos ilocucionarios y actos mágicos», en Crítica jurídica. Revista Latinoamericana de Política, Filosofía y Derecho, Núm. 13, 1993, pp. 195-225.

- «The Functioning of Human Rights in the Legal System», en Arnau, J.A., Hilpinen, R. y Wroblewski, (Eds.), Juristische Logik, Rationalität und Irrationalität im Recht, en Rechtstheorie, Berlin/Mainz, Verlag Duncker \& Humblot, 46 Band, Beheif 8, 1985, pp. 375-386.

- «The Inner Logic of Law and Legal Hermeneutics», en Eckhoff, T., Friedman, L.M. y Uusitalo, J., (Eds.), Vernunft und Erfahrung im Rechtsdenken der Gegenwart, en Rechtstheorie, Berlin /Mainz, Verlag Dunker \& Humblot 46. Band, Beheif 10, 1986, pp. 119-123.

TWINING, WILLIAM Y MIERS, DAVID, How to do Things with Rules, Weidenfeld and Nicolson, Londres, 1991.

VERNENGO, ROBERTO, «La interpretación lógica del derecho y formas de decisión» en Anuario de Filosofía Jurídica y Social, Núm. 9, 1989, pp. 195-220. Buenos Aires.

- Lógicas normativas y la reconstrucción del razonamiento jurídico, Vol. VII, Nos. 16-18, 1992, pp. 1115-1124.

WALKER, DAVID M., Oxford Companion to Law, Oxford, Oxford University Press, 1980.

WEINBERGER, OTTA, «The Role of Rules», en Ratio Iuris, Núm. 3, pp.?

WRIGHT, G. H. VON, Norm and Action, Routledge and Kegan Paul, Londres, 1963.

- «Is there a Logic of norms?» en Ratio Juris, Núm. 4, 1991, pp. 265-283.

ZACCARIA, G., L'Arte dell'interpretazione. Saggi sull'ermeneutica giuridica contemporanea, Padua, Cedam, 1990. 